\title{
Usability - Not as we know it!
}

\author{
Paula Alexandra Silva \\ Lancaster University \\ Computing/Infolab21 \\ Lancaster, LA1 4YR, UK \\ +441524510319 \\ palexa@gmail.com
}

\author{
Alan Dix \\ Lancaster University \\ Computing/Infolab21 \\ Lancaster, LA1 4YR, UK \\ $+441524510319$ \\ alan@hcibook.com
}

\begin{abstract}
YouTube has been the Internet success story of 2006. However, when subjected to conventional usability evaluation it appears to fail miserably. With this and other social Web services, the purpose of the user is fun, uncertainty, engagement and selfexpression. Web2.0 has turned the passive 'user' into an active producer of content and shaper of the ultimate user experience. This more playful, more participative, often joyful use of technology appears to conflict with conventional usability, but we argue that a deeper 'usability' emerges that respects the user's purposes whether acting as homo ludens.
\end{abstract}

\section{Categories and Subject Descriptors}

H.5.5 [Information Interfaces and Presentation]: HCI

\section{General Terms}

Design, Human Factors

\section{Keywords}

YouTube, user experience, Web2.0, Evaluation, Design

\section{INTRODUCTION}

YouTube was last year's Web success story. A website that allows users to store and share personal videos, subsequently, linked, searched and/or rated, has gone from zero to $60 \%$ of all online viewing in just 18 months [1]. Its huge and sudden popularity made it the Internet phenomenon of 2006.

However, when analysed with conventional usability heuristics it fails many. So is the popularity in spite of this - the draw of the content meaning that YouTube can afford poor usability? Or is it more fundamental, conventional usability neglects or conflicts with the more ludic aims of the site? We have become used to debates or even argument between user experience design and more Taylorist usability engineering. However, Web2.0 sites, such as YouTube, add more complexity to this picture as the user becomes the producer.

In this paper, we explore some of these issues, first analysing YouTube from a conventional usability standpoint and then exploring the reasons why this does not give a true indication of the real 'usability' of the system and how conventional conceptions may need to change... and where they do not.

Paula Alexandra Silva and Alan Dix, 2007

Published by the British Computer Society

Volume 2 Proceedings of the 21st BCS HCI Group Conference

HCI 2007, 3-7 September 2007, Lancaster University, UK Devina Ramduny-Ellis \& Dorothy Rachovides (Editors)

\section{THE YOUTUBE PHENOMENON}

YouTube became the Internet phenomenon of 2006, in the sense of a site, idea or arguable 'meme' that spreads with extreme speed due to the size and/or often social interconnectedness of the internet [9].

According to Alexa's website ${ }^{1}$, YouTube is the fourth most visited website, and the first if we do not include search engines (see Figure 1). Also, if we look at the Web ratings by country, we realise that YouTube is also quite popular, for instance, it is the $5^{\text {th }}$ most accessed site in the US, Portugal and Spain, the $6^{\text {th }}$ in Canada and Japan and the $8^{\text {th }}$ in the UK.

\begin{tabular}{|c|c|c|}
\hline \multicolumn{3}{|c|}{ Alexa Top Sites } \\
\hline Rank & Change & Web Site \\
\hline 1 & (none) & yahoo.com \\
\hline 2 & (none) & msn.com \\
\hline 3 & (none) & google.com \\
\hline 4 & $\Delta 1$ & youtube.com \\
\hline 5 & $\Delta 1$ & myspace.com \\
\hline 6 & $\Delta 1$ & live.com \\
\hline 7 & 8 & baidu.com \\
\hline 8 & (none) & orkut_com \\
\hline 0 & $\Delta n$ & Wrilkinadin are \\
\hline
\end{tabular}

Figure 1: Alexa top sites (22-05-2007)

YouTube is the type of site a user accesses when he or she has some spare time, and just feels like 'hanging around' the Web, or when someone, usually a friend or colleague, sends him or her a link to an interesting video. So, we are here referring to a particular kind of public that includes Web surfing among its hobbies and that, in some way, socialises via the Web.

These phenomena have been recognised in other Web services, such as MySpace, Blogger or Flicker. Interestingly, these sites obey the $1 \%$ rule: for each 100 people online, just 1 will create content, 10 will interact ${ }^{2}$ with it and the remaining 89 will only view it. For YouTube, each day, there are 100 million downloads and 65,000 uploads, that is 1,538 downloads per upload (Antony Mayfield cited in Arthur [1]).

Finally, placing ourselves in the role of Web designers and/or evaluators, when we consider YouTube from a usability pointof-view, it seems poorly designed, or maybe has no design at all: just a chaotic, cluttered website. However, as we

\footnotetext{
${ }^{1}$ Alexa (www.alexa.com) provides information on web traffic to other websites collected from users of Alexa Toolbar.

${ }^{2}$ Interact here includes commenting or contributing somehow with improvements.
} 
understand this phenomenon we find that, maybe, it is not at all a case of bad design. This leads us to reflect: What is changing in Web interaction? What type of user are we now dealing with? If the Web has adopted new roles? What must consequentially change in web evaluation and (re)design?

\section{EVALUATING YouTube}

How do we evaluate YouTube? How would YouTube perform if we evaluate it according to conventional usability metrics? In this section, we will try to answer these questions, by reflecting on and providing some examples of usability problems, as well as giving our own thoughts and suggestions about it.

\subsection{Conventional YouTube evaluation}

One of the most common, popular and advantageous usability evaluation techniques is Heuristic Evaluation [2, 3], which allows experts to critique an interface by verifying its agreement with general and simple heuristics ${ }^{3}$ or principles, such as clarity, consistency or fluidity of navigation. Following the list of the ten Nielsen's recommended heuristics $[2,8]$ for a usable interface design we can audit YouTube's usability. Our general evaluation is shown by a $\uparrow-$ positive, $\downarrow-$ negative, $\leftrightarrow$ - present but unsatisfactory.

Visibility of system status $-\uparrow-$ Concerning this guideline, YouTube performs generally well, as it keeps the user informed about what the system is doing, by providing information about loading and total video times.

Match between system and the real world $-\leftrightarrow-$ This heuristic verifies to what extent the language of the interface respects the user's familiar language and concepts. On the one hand the video controls, similar to domestic video players, respect real-world conventions, but on the other hand, there is a vast amount of unclear terminology and associated functions, such as: quicklists, featured videos or channel names ${ }^{4}$.

User control and freedom $-\uparrow-$ This is the principle in which YouTube performs the best. In fact, the use of the conventional video commands allows full control and freedom to the user who then uses the interface skilfully.

Consistency and standards $-\downarrow-$ In contrast, this is where YouTube performs worst. Two examples are: i) Navigation history is lost when the user logs into the system, so, for instance, the loss of videos watched, quicklists, etc; and ii) Comments are frequently chats between users, but as these are not presented over a linear, dated logic, the context and understanding are mislaid.

Error prevention $-\downarrow-$ This guideline is not observed; for example, when watching a video in a channel page, a table of contents is shown, although every time the user clicks in any of the other videos, the user is directed to a standard page losing any previous contextual information. In order to re-access it, being obliged to use the back button. The same happens when you are visualizing the comments and several other basic tasks.

Recognition rather than recall $-\downarrow$ - Interfaces should not rely on user's memory, making actions visible to the user. Interfaces

\footnotetext{
${ }^{3}$ A heuristic is a guideline, general principle, or rule that describes properties of a usable interface. It can be used to guide the design or to critique a decision $[2,7,8]$.

${ }^{4}$ Channel titles are not always meaningful. For instance, names as brokensonnet 2 are probably helpless for a novice user, and therefore will not help on deducting what is under a channel with such name.
}

should not rely on the user's memory, by making actions immediately visible to the user. Besides the not obvious terminology, the page's layout on lower resolution screens requires the user to navigate with the scroll bar to vital components such as the search box. These are often fundamental if the user is purposely looking for specific content on the site, instead of following a link sent by a friend or lingering around. This requires the user to recall the position or location of such functions, instead of merely recognizing them.

Flexibility and efficiency of use $-\leftrightarrow-$ The user has full mastery of the video controls, but there are various functions in which the system performs poorly. An important example refers to the retrieved search results that cannot be ordered or organised under any criteria, such as date or rating.

Aesthetic and minimalist design - $\downarrow$ - If we consider the quantity of images, videos and other types of information available on YouTube pages, we are temped to state that this heuristic is simply not considered. In fact, the first general reaction to YouTube pages is of chaos.

Help users recognize, diagnose, and recover from errors $\leftrightarrow-$ This heuristic gives direction on how to express error messages. These are only present when the user is signing up.

Help and documentation $-\leftrightarrow-$ While, arguably, a system should be usable without documentation, help and documentation are necessary! YouTube has FAQ-style help, but less in terms of 'how-to do it' documentation.

In summary, from the list of ten recommended Nielsen's heuristics, YouTube respects only two, with the rest failing or unsatisfactory. From these results, we can say that YouTube appears to fail miserably when evaluated with a conventional usability evaluation technique. Moreover, if we consider the most precious usability metrics, total task completion time, total number of clicks and total number of errors, YouTube, definitely fails. However, YouTube's clear success means there must be something really good that makes users go back and back again.

\subsection{YouTube evaluation - not as we know it}

Having examined how it fails, we now consider why YouTube succeeds focusing on the website purpose, content, and design.

\subsubsection{Reasons for success}

As we demonstrated in section 3.1, YouTube is a disaster from a conventional usability evaluation point-of-view. Thus, users were not expected to return to reuse the system, and definitely not to make it such as show-case of success. So what are the reasons for its success? We identified three possible answers: its users, its content and its design.

\section{Users}

Current Web users are very different from those of the '90's. We now have also a generation of users that grew up on and together with the Web and its technology. Therefore they are reasonably literate in all that concerns the Web, its tools, services, etc, and use them just as any other artefact in their everyday life. Additionally, they access YouTube because they want to or just happen to have some free time. Finally, partly as a consequence of the latter, these users are content designers themselves, communicating and socialising via the Web.

\section{Content}

YouTube content is largely responsible for YouTube success. First there is the aspect of personal broadcasting of a user that wants to communicate and extend him or herself via the web. In fact, there is not unusual to find users simply broadcasting their 
daily routines, as a kind of digital diary, or their participation in a conference. These are then shared with a vast community of friends, relatives or anyone potentially accessing the Web. Then, and being particularly relevant, we have all the amateur videos as well as movies and television series that have not yet being released. These videos are sometimes illegal.

\section{(Apparent bad) Design}

Without forgetting any of the faults reported in section 3.1, we now emphasise specifically four of them - aesthetic and minimalist design, error prevention, total number of clicks and task completion time - because, as we will explain, these may, in fact, constitute the smart features of the YouTube interface.

We will start by explaining why task completion time is so relevant for YouTube, but from a totally opposite perspective to the one of the past! In fact, as YouTube is mainly supported by advertisement the longer we keep the user hanging out the better, so it is important for users to be able to accomplish their tasks but not in the shortest possible time. For the same reason, the total number of clicks should not be reduced to a minimum, because the more pages the user browses, the more he or her is likely to be subjected to more ads!

The error prevention is another form, almost cruel, of keeping the user in the system. In effect, the user is permanently losing the interaction context, and forced to use the back button, or, if unlucky, restart the navigation.

Finally, concerning aesthetic and minimalist design, we need only to consider the major and permanent information clutter. Image and text chaos is evident when we access and browse YouTube (see Figure 2 and Figure 3).

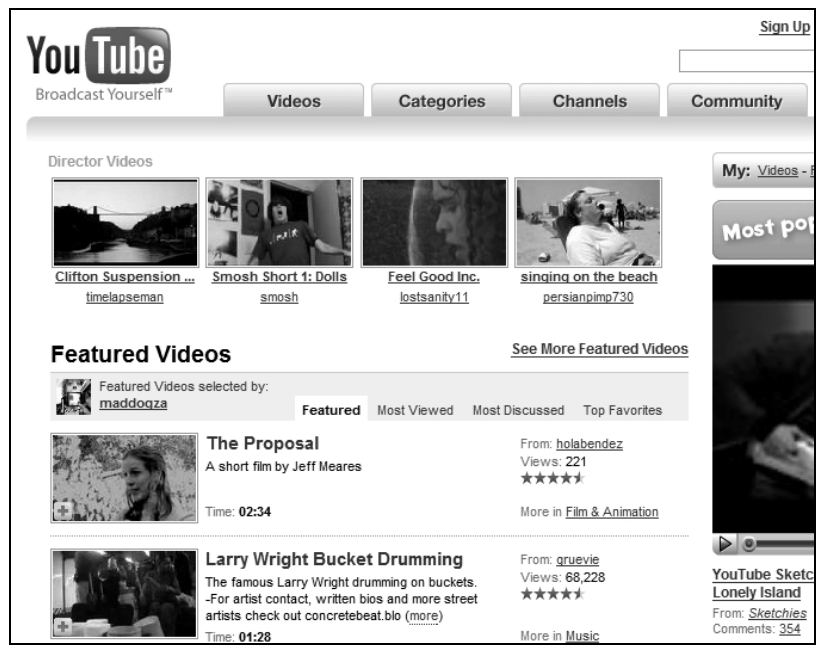

Figure 2: YouTube main page

To comprehend this, we recall Miller's [6] magical number seven and Hicks law ${ }^{5}$ [4]. How many chunks of information does a user have to deal with when browsing YouTube? How many choices does the user have available in each YouTube page? Certainly, these two metrics are large and, inevitably make demands on the human perception and reasoning systems. Various images concurrently catch the attention of user that

\footnotetext{
${ }^{5}$ This law describes the time it takes for a user to make a decision. Given $n$ equally probable choices, the average reaction time $T$ required to choose among them is approximately $T=b \log _{2}(n+1)$ where $b$ is a constant that can be determined empirically by fitting a line to measured data (for a particular individual and context).
}

clicks one and another image and, interminable and subsequently, watches the videos associated with them. The pace is defined by how fast the user's eye is captured by a different image and the user's attention and interest are released from the previous video.

YouTube success has proven that none of the (apparently) bad design features here presented have prevented the increasing use of YouTube; indeed, we think they have favoured it, being responsible for it. So, we wonder: How would YouTube be if "well" designed? How would it perform? Would it still achieve its purpose? Would it yet be a success?

\subsubsection{Crucial features}

Having presented the possible reasons for YouTube success, we now reflect on the interface features contributing for this intriguing interaction experience.

\section{User control and freedom}

The design features discussed above might not have survived, nor indeed YouTube itself, if they were not balanced by an excellent interaction feature - user full sense of control and freedom of the system. This matter is well achieved by the YouTube interface and is extremely important from a user interaction point-of-view. The conventional video controls, similar to domestic video players, allow users full control and freedom when interacting with the system. It is easy to choose a video just by looking at its image, title and short description. But also, if the video does not correspond to the user expectations, it is equally easy to immediately choose and change to another video among the nearest bit of clutter.

Interaction in YouTube is as easy and similar to the use of any common remote control while zapping through your endless television channels. Another similarity with television zapping is the "syndrome of the unfinished movie"; we always think we should watch it a bit longer just in case it gets more interesting or it has a surprising ending. So we keep on prolonging our experience expecting something better to appear.

\section{Engagement}

Another wise feature of YouTube is related to the way the system manages to keep the user engaged with the system. This is achieved as follows. First, when the user is accessing the main YouTube page (Figure 2), he or she is helped in the selection of videos by visual clues to: i) featured, ii) most viewed, ii) most discussed; and iv) top favourites videos.

Then, after having selected a video and when in a YouTube video viewing page (Figure 3); ie: before, while and after watching the video, the service provides a set of information about the video such as i) related, ii) more from this user, and iii) playlists or i) about the video, ii) rating, iii) views, iv) comments and v) favorited. These seduce users' curiosity and tempts them to jump from one movie to another and yet another. This creates a cycle that together with unexpectedness and sense of surprise creates a remarkable engagement.

\section{The homo ludens}

In the 1950's, Huizinga coined the term Homo Ludens [5], defining humans as playful creatures and suggested play was crucial to the generation of culture. Play includes acting, games, fun - activities that require human action (maybe performance) and engagement. YouTube clearly addresses this basic human need and indeed is rapidly creating its own cultural shifts.

Huizinga particularly emphasises that play is for itself and of all activities most about freedom - just because it is superfluous 
(see [5] pp.7, 8). However, he also shows how play permeates the 'serious' business of life from law to war, from poetry to philosophy. As well as just 'having a good time', the freedom of the 'playful' element in YouTube allows users to express (or portray) themselves and do the serious work of identity and sociality.

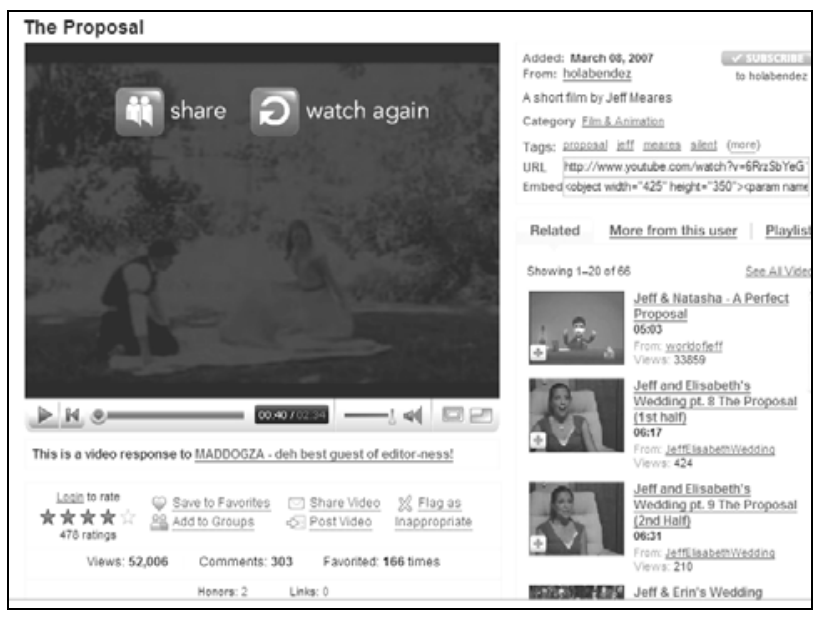

Figure 3: YouTube video viewing page

\section{SO DOES USABILITY MATTER?}

If YouTube violates so many usability principles and yet is still so successful, does usability matter at all, especially for the channel-hopping, iPod-shuffling generation brought up with pervasive technology and incessant media?

\subsection{Fitness for Purpose}

Usability is always part of a broader agenda of designing things that are fit for purpose. There are always conflicts here, for example we often trade usability for security. There are also conflicts between fitness for purpose for a user and for the supplier, for example, Amazon forbids reviews with URLs to avoid links to rivals - good for the user but bad for Amazon.

In traditional GUI systems the users' purpose is assumed to be to attain some goal and the purpose of the designer to help them do so. Usability guidance, such as Nielsen's Heuristics, embodies this assumption. In YouTube the users' purpose is explore, have fun, and enjoy the 'route' as much as the goal. Not surprisingly 'usability' guidance should change accordingly.

\subsection{Designers and Users}

YouTube has two (overlapping) classes of end users: those who browse and those who produce content. In 'producer' role, their purpose and goals are closer to traditional systems - to upload a video, change some setting. Here traditional usability principles still apply and indeed in recent discussions with a group of relatively computer-naïve end-users, they cited YouTube's instructions for video upload as an exemplar of good practice. Similarly when a browsing user wants something specific: to subscribe to a video producer or look in their history for a recently seen video - here again conventional advice applies.

More problematically YouTube and such sites have two (potentially non overlapping) classes of designers: the professionals and the end-users configuring their own pages (the second class of users above). The purpose of the second class, the user-as-designer, includes self-expression, publicizing videos, maybe directing visitors towards specific content. The purpose of the 'professionals', is then both to make it easy for user-as-designer and for the result to be fit for purpose for the browsing users. In YouTube the freedom of the user - as designer - is relatively limited, so the browsing users have a similar structure across the site. In other Web2.0 sites such as MySpace, this becomes a more major issue - how do you create frameworks that allow expression and yet have some level of overall usability (in its broadest sense)... and how do you help (or educate?) users-as-designers to use these most effectively.

\section{DISCUSSION AND FURTHER WORK}

A more detailed study about YouTube-like websites, such as Wikipedia, Flicker, Blogger or MySpace, would allow us to validate some of our assumptions, for instance with respect to the reasons why a user accesses this type of services and for how long? Furthermore, information gathered would permit a better understanding of these Web Phenomena, its services, purposes, design and evaluation. So far, the similarities we identify make us conclude that the Web is approaching or already going through a new stage, in which the increasing number of young but literate web users will play a major role. This user wants and is able to manipulate playful, participative and joyful Web services. This new context requires new and sharp usability evaluation approaches that we, as researchers and/or academics, should include in our repertoire. From this study we learnt that the user's sense of control and freedom, engagement and playfulness are critical qualities for success.

\section{REFERENCES}

[1] Arthur, C.. What is the 1\% rule? The Guardian, July 20 , 2006 (accessed 22-01-2007) http://technology.guardian.co.uk/weekly/story/0,,1823959, 00.html

[2] Dix, A., Finlay, J., Abowd G. and Beale, R. HumanComputer Interaction. Pearson/Prentice-Hall, 2003.

[3] Ellis, P. and Ellis, S. Measuring User Experience. 2001 http://www.webtechniques.com/archives/2001/02/ellis/

[4] Hick, W. On the rate of gain of information. Quarterly Journal of Experimental Psychology, 4 (1952), 11-26.

[5] Huizinga, J. Homo Ludens: A Study of the Play Element in Culture. Boston: The Beacon Press, 1950

[6] Miller, G. The Magical Number Seven, Plus or Minus Two: Some Limits on Our Capacity for Processing Information. The Psychological Review, 63 (1956), 81-97.

[7] Nielsen, J. Usability Engineering. USA, Morgan Kaufmann Publishers, Inc., 1993

[8] Nielsen, J. Heuristic Evaluation. useit.com. (accessed 12/04/2000) http://www.useit.com/papers/heuristic/. Please see also related links.

[9] http://en.wikipedia.org/wiki/Internet_phenomena (accessed 22-01-2007) 